\title{
Ramsay-Hunt syndrome in the differential diagnosis of stroke
}

\section{Alexandre Costa ${ }^{[1]}$ and Andreia Veiga ${ }^{[1]}$}

[1]. Departamento de Neurologia, Centro Hospitalar de Trás-os-Montes e Alto Douro, Vila Real, Portugal.

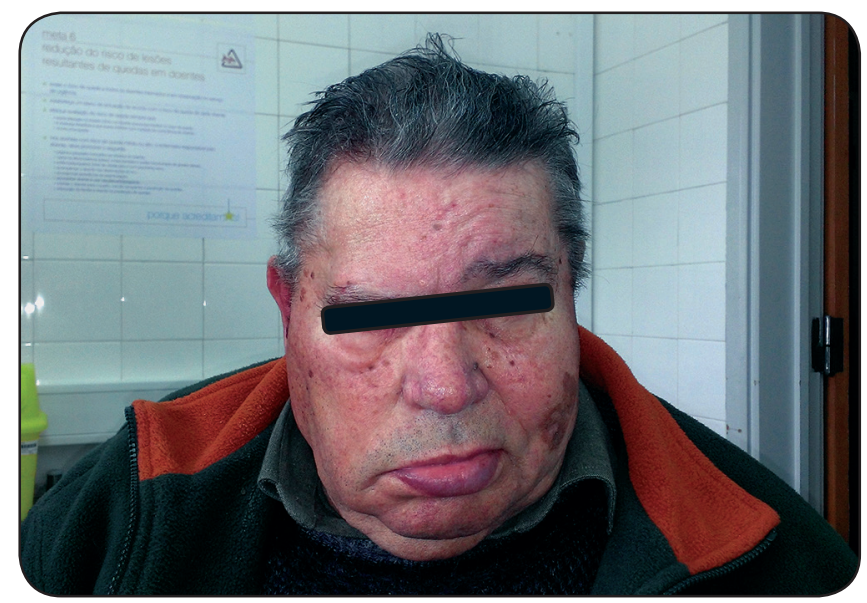

(A)

An 82-year-old man with a medical history of diabetes, hypertension, and right hemispheric stroke 4 years previously, presented to the emergency department complaining of pain in the right ear, otorrhea, and hypoacusis for 5 days. His spouse also mentioned symptoms of drooling and aggravation of previous deficits, namely dysarthria, facial asymmetry, and left hemiparesis. Due to the suspicion of a new stroke event, a neurological evaluation was requested, which revealed right peripheral facial palsy (Figure A), ipsilateral hypoacusis, and spastic left hemiparesis with impaired deambulation without support. An ear inspection revealed various blistering lesions and cicatricial tissue on the right external auditory canal (Figure B). Routine blood analysis was normal, and cerebral computed tomography showed no recent lesions, particularly ischemic or hemorrhagic lesions. The cerebrospinal fluid investigation revealed pleocytosis $(75$ cells, $95 \%$ of which were mononuclear), normal glucose and protein values, and negative results for the microbiological investigation, including unsuccessful isolation of varicella zoster virus (VZV). The patient was treated with acyclovir (800mg 5 times daily) and

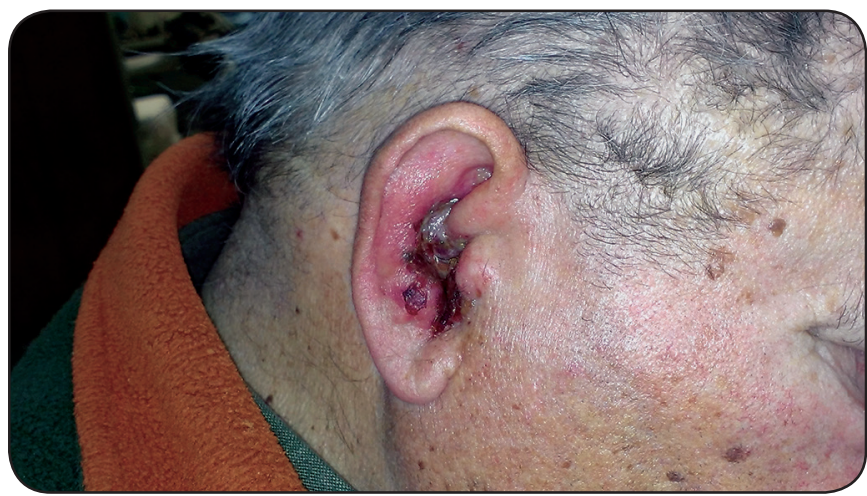

(B)

prednisone (50mg daily) for 5 days and submitted to an urgent rehabilitation program. After 3 months of follow-up, the patient demonstrated residual improvement but maintenance of severe right facial paralysis.

Ramsay-Hunt syndrome is caused by the reactivation of latent VZV infection in the geniculate ganglion of the facial nerves ${ }^{1}$. This diagnosis is generally clinical, although a positive polymerase chain reaction (PCR) test for VZV is used for confirmation ${ }^{2,3}$. The current case illustrates how previous neurological deficits can confound an apparently straightforward diagnosis and underscores the need to consider different diagnoses.

\section{REFERENCES}

1 Kansu L, Yilmaz I. Herpes zoster oticus (Ramsay Hunt syndrome) in children: case report and literature review. Int J Pediatr Otorhinolaryngol 2012; 76:772-776.

2 Sweeney CJ, Gilden DH. Ramsay Hunt syndrome. J Neurol Neurosurg Psychiatry 2001; 71:149-154.

3. Wagner G, Klinge H, Sachse MM. Ramsay Hunt syndrome. JDDG 2012; 10:238-243.

Address to: Dr. Alexandre Costa. Av. da República 1583 Hab. 8, Vila Nova de Gaia, Portugal.

Phone: 35125 930-0574; Fax: 35125 930-0589

e-mail: alxjscosta@gmail.com

Received 22 August 2013

Accepted 20 September 2013 